\title{
Evidence for Disk Truncation at Low Accretion States of the Black Hole Binary MAXI J1820+070 Observed by NuSTAR and XMM-Newton
}

 \\ ${ }^{1}$ Cahill Center for Astronomy and Astrophysics, California Institute of Technology, Pasadena, CA 91125, USA \\ ${ }^{2}$ Space Sciences Laboratory, 7 Gauss Way, University of California, Berkeley, CA 94720-7450, USA \\ NASA Goddard Space Flight Center, Greenbelt, MD 20771, USA \\ ${ }^{4}$ Institute of Astronomy, University of Cambridge, Madingley Road, Cambridge CB3 0HA, UK \\ Received 2020 January 26; revised 2020 March 2; accepted 2020 March 3; published 2020 April 14
}

\begin{abstract}
We present results from NuSTAR and XMM-Newton observations of the new black hole X-ray binary MAXI J1820 +070 at low accretion rates (below $1 \%$ of the Eddington luminosity). We detect a narrow $\mathrm{Fe} \mathrm{K} \alpha$ emission line, in contrast to the broad and asymmetric $\mathrm{Fe} \mathrm{K} \alpha$ line profiles commonly present in black hole binaries at high accretion rates. The narrow line, with weak relativistic broadening, indicates that the $\mathrm{Fe} \mathrm{K} \alpha$ line is produced at a large disk radius. Fitting with disk reflection models assuming standard disk emissivity finds a large disk truncation radius (a few tens to a few hundreds of gravitational radii, depending on the disk inclination). In addition, we detect a quasi-periodic oscillation (QPO) varying in frequency between $11.6 \pm 0.2 \mathrm{mHz}$ and $2.8 \pm 0.1 \mathrm{mHz}$. The very low QPO frequencies suggest a large size for the optically thin Comptonization region according to the Lense-Thirring precession model, supporting that the accretion disk recedes from the innermost stable circular orbit and is replaced by advection-dominated accretion flow at low accretion rates. We also discuss the possibility of an alternative accretion geometry that the narrow $\mathrm{Fe} \mathrm{K} \alpha$ line is produced by a lamppost corona with a large height illuminating the disk.
\end{abstract}

Unified Astronomy Thesaurus concepts: Accretion (14); Black hole physics (159); X-ray transient sources (1852); X-ray binary stars (1811)

\section{Introduction}

Low-mass black hole X-ray binaries contain stellar-remnant black holes accreting from donor stars with a mass of $<1 M_{\odot}$ that transfer mass by Roche lobe overflow. Most known Galactic black hole X-ray binaries are low-mass X-ray binaries and are discovered as transients. These systems exhibit recurrent outbursts on year to decade timescales, during which their flux increases by several orders of magnitude in the optical and X-ray bands (e.g., Corral-Santana et al. 2016). During a typical outburst, lasting for a few months, a black hole binary transitions between different X-ray spectral states, and displays distinct X-ray spectral and timing properties (see Remillard \& McClintock 2006 for a review).

MAXI J1820+070 (=ASASSN-18ey) is a new transient black hole X-ray binary discovered in 2018. The outburst was first reported in optical by the All-Sky Automated Survey for SuperNovae on UT 2018 March 06.58 (ASAS-SN; Tucker et al. 2018), and subsequently in the X-ray band by MAXI a week later (Kawamuro et al. 2018). The source reached a peak X-ray luminosity (2-20 keV) of about $2 \mathrm{Crab}$, becoming one of the brightest X-ray novae discovered. Its outburst was well covered by multiwavelength observations from the radio to the gamma-ray bands (e.g., Bright et al. 2018; Shidatsu et al. 2018; Hoang et al. 2019; Paice et al. 2019). The distance to MAXI $\mathrm{J} 1820+070$ is estimated as $5.1 \pm 2.7 \mathrm{kpc}$ or $4.4 \pm 2.4 \mathrm{kpc}$ (Atri et al. 2020), and $3.46_{-1.03}^{+2.18} \mathrm{kpc}$ (Gandhi et al. 2019) based on different distance priors from the Gaia Data Release 2 parallax (Gaia Collaboration et al. 2018). From radio parallax, the distance is measured as $2.96 \pm 0.03 \mathrm{kpc}$ (Atri et al. 2020). MAXI J1820+070 is recently dynamically confirmed as a black hole binary (BHB) accreting from a K3-5 type donor star

\footnotetext{
${ }^{5}$ NASA Postdoctoral Program Fellow.
}

with a 0.68 day orbit, and the mass estimate of the central black hole is 7-8 $M_{\odot}$ (Torres et al. 2019). Due to its high X-ray flux, MAXI J1820+070 is an ideal new target for the study of the inner accretion flow properties around black holes via X-ray spectral and timing analyses. X-ray timing analysis of observations by the Neutron star Interior Composition Explorer (NICER) provides clues about the evolution in the coronal geometry (Kara et al. 2019). NuSTAR observations of MAXI $\mathrm{J} 1820+070$ during the bright phases of the hard state display relativistic disk reflection features, including a broad $\mathrm{Fe} \mathrm{K} \alpha$ line that is nearly invariant in the line profile over multiple observations at different fluxes. Detailed modeling of the reflection spectra reveals that the inner edge of the accretion disk remains stable at about 5 gravitational radii, which indicates that the central black hole is likely to have a low spin (Bharali et al. 2019; Buisson et al. 2019). In addition, a lowfrequency quasi-periodic oscillation (QPO) varying in frequency is detected in MAXI J1820+070 in both optical and X-ray bands (e.g., Yu et al. 2018; Buisson et al. 2019).

Black hole accretion is generally studied in two regimes: cold accretion flows of optically thick material at high mass accretion rates; and optically thin hot accretion flows at low accretion rates. The latter is thought to contain an advection-dominated accretion flow (ADAF and its variants, see Yuan \& Narayan 2014 for a review). The ADAF model explains the hard X-ray emission from black holes accreting at low accretion rates, e.g., BHBs in quiescence and low/hard states, low luminosity active galactic nuclei (LLAGNs), and also Sgr A*. In BHBs, the accretion geometry is deduced to be in the form of an optically thick accretion disk at large disk radii, which evaporates and is replaced by ADAF close to the black hole (e.g., Esin et al. 1997). The transitional radius, or the truncation radius of the optically thick accretion disk, is predicted to increase with decreasing accretion rate (e.g., Meyer et al. 2000; Taam et al. 2012). 


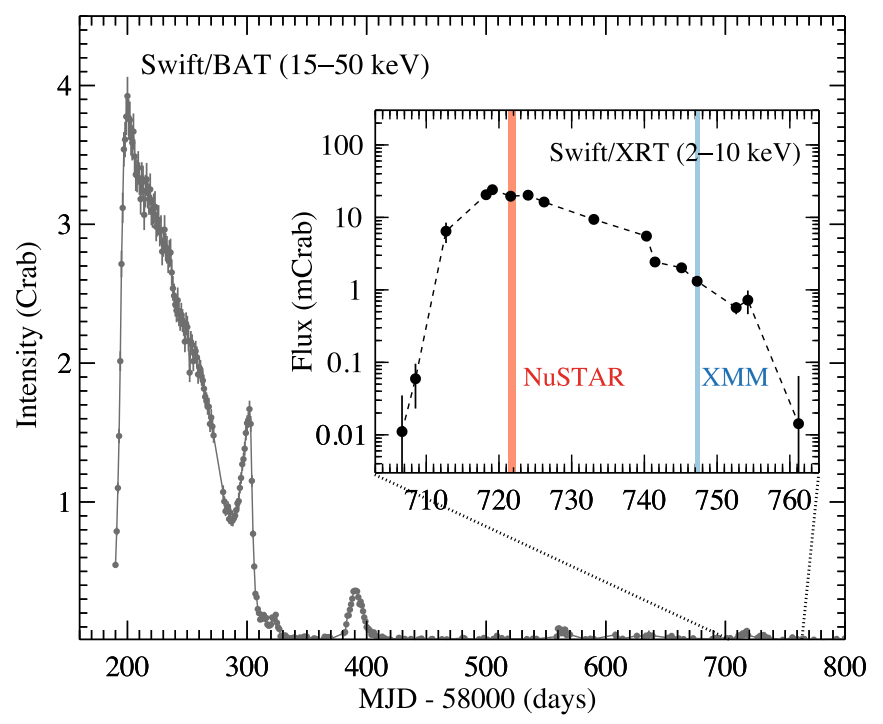

Figure 1. Long-term Swift/BAT monitoring light curve of the outburst of MAXI J1820+070. The BAT count rate is divided by the Crab count rate. The insert is the X-ray flux of MAXI J1820+070 measured by Swift/XRT close to the rebrighting period. The shaded areas mark the duration of the NuSTAR and XMM-Newton observations analyzed in this work.

Observationally, the inner radius of the accretion disk in BHBs can be measured by modeling the strength and temperature of the thermal disk emission component (Zhang et al. 1997), or modeling the profile of $\mathrm{Fe} \mathrm{K} \alpha$ emission line that originates from reflection of hard X-ray photons from the corona by the accretion disk (Fabian et al. 1989). The line profile becomes broad and asymmetric, with an extended red wing originating from the vicinity of the black hole due to combinational effects of Doppler shift, relativistic beaming and gravitational redshift (see Miller 2007 for a review). Recent observations of several bright BHBs by $\mathrm{NuSTAR}$ revealed clearly broad $\mathrm{Fe} \mathrm{K} \alpha$ lines, indicating that the accretion disk extends all the way down to the innermost stable circular orbit (ISCO) in the bright hard state (BHS; e.g., Miller et al. 2015; Xu et al. 2018a, 2018b; Buisson et al. 2019). In the low hard state (LHS), the detection of a narrow $\mathrm{Fe} \mathrm{K} \alpha$ line, the evidence for disk truncation, is usually hindered by the faintness of the targets, the weakness of the reflection features, and the limited instrumental spectral resolution. So far, narrow $\mathrm{Fe} \mathrm{K} \alpha$ emission has only been convincingly detected in the BHB candidate GX 339-4 from a long Suzaku observation taken in 2008 (Tomsick et al. 2009).

\section{Observation and Data Reduction}

We triggered NuSTAR (Harrison et al. 2013) and XMMNewton (Jansen et al. 2001) observations of MAXI J1820+070 during the second rebrightening period after the the end of its 2018 main outburst (Xu et al. 2019). During this rebrightening period, the source stayed in the LHS at a low accretion rate (below $1 \%$ of the Eddington limit), but reached an X-ray flux level high enough to enable the detection of the $\mathrm{Fe} \mathrm{K} \alpha$ emission line.

We show the time of the NuSTAR and XMM-Newton observations in the Swift/Burst Alert Telescope (BAT; Barthelmy et al. 2005) and X-ray Telescope (XRT; Burrows et al. 2005) monitoring light curves of MAXI J1820+070, see Figure 1. The Swift/BAT light curve was obtained from the Swift/BAT Hard X-ray Transient Monitor ${ }^{6}$ (Krimm et al. 2013). We reduced the

\footnotetext{
6 https://swift.gsfc.nasa.gov/results/transients/
}

Swift/XRT data using xrtpipeline v0.13.5 with CALDB v20190910. We extracted source spectra from a circular region with a radius of $60^{\prime \prime}$, and the background was extracted from an annulus with inner and outer radii of $160^{\prime \prime}$ and $300^{\prime \prime}$. The X-ray flux in $2-10 \mathrm{keV}$ measured by XRT was estimated by fitting the spectra in the energy range of $0.3-10 \mathrm{keV}$ with an absorbed power-law model.

\subsection{NUSTAR}

NuSTAR observed MAXI J1820+070 on 2019 August 26 starting from UT 07:16:09 (ObsID: 90501337002). We reduced the data using NuSTARDAS pipeline v1.8.0 and CALDB v20191008. The source spectra were extracted from a circular region with the radius of $180^{\prime \prime}$ from the two focal plane modules (FPMA and FPMB). Corresponding background spectra were extracted using polygonal regions from sourcefree areas. We also extracted spectra from mode 06 data to maximize the available exposure time following the method outlined in Walton et al. (2016). The resulting exposure times are $48.6 \mathrm{ks}$ and $48.4 \mathrm{ks}$ for FPMA and FPMB, respectively. We coadded the FPMA and FPMB spectra using the addspec tool in HEASOFT. The NuSTAR spectra were grouped to have a signal-to-noise ratio $(\mathrm{S} / \mathrm{N})$ of at least 10 per bin. We applied barycenter corrections to the event files, transferring the photon arrival times to the barycenter of the solar system using JPL Planetary Ephemeris DE-200, and extracted source light curves from the same region as the energy spectra.

\subsection{XMM-Newton}

The XMM-Newton observation of MAXI J1820+070 (ObsID: 0851181301) started on 2019 September 20 from UT 22:45:46. Data reduction is performed using the XMMNewton Science Analysis System v17.0.0 following standard procedures. EPIC-MOS1, MOS2 and EPIC-pn operated in the timing mode, but EPIC-MOS1 experienced full scientific buffer during the whole observation. EPIC-pn (Struider et al. 2001) is the prime instrument we use due to its large effective area in the Fe K band. The net exposure time of EPIC-pn is $56 \mathrm{ks}$ after filtering out periods of high background flaring activity. The data is free from pile-up effects at a mean count rate of $\sim 20$ counts $\mathrm{s}^{-1}$. We selected events with pattern $\leqslant 4$ (singles and doubles) and quality flag $=0$. The source spectrum and light curve were extracted from the columns of $27 \leqslant$ RAWX $\leqslant 47$, and the corresponding background was extracted from $58 \leqslant$ RAWX $\leqslant 60$. We used rmfgen and arfgen to generate the redistribution matrix files and ancillary response files. We grouped the EPIC-pn spectrum to have a minimum $\mathrm{S} / \mathrm{N}$ of 10 for spectral modeling. The collected source light curve was barycenter corrected using the barycen tool, and corrected for instrumental effects by epiclccorr.

\section{Spectral Analysis}

In this work, we perform all spectral modeling in XSPEC v12.10.1f (Arnaud 1996). We use the cross sections from Verner et al. (1996) and abundances from Wilms et al. (2000) in the TBabs neutral absorption model. All uncertainties are reported at the $90 \%$ confidence level unless otherwise clarified. We fit NuSTAR spectra from 3.5-79 keV, and XMM-Newton EPIC-pn spectrum from $0.6-10 \mathrm{keV}$.

We first model the NuSTAR (=EPOCH 1) and XMMNewton (=EPOCH 2) spectra with an absorbed power-law 



Figure 2. Top: NuSTAR (EPOCH 1, left) and XMM-Newton (EPOCH 2, right) spectra of the MAXI J1820+070 in the LHS, unfolded with the best-fit model. The coronal emission directly observed and the disk reflection component are plotted in dashed and solid lines, respectively. Middle and bottom: data/model spectral residuals. The spectra are rebinned for display clarity. The dips in the NuSTAR spectrum at about 11 and $26 \mathrm{keV}$ and the excess in the XMM-Newton EPIC-pn spectrum around $8 \mathrm{keV}$ are calibration related.

model, TBabs*powerlaw, in XSPEC. The NuSTAR and XMM-Newton observations were taken in two epochs separated by 25 days, thus we fit the spectra individually with no linked parameters. We freeze the absorption column density, $N_{\mathrm{H}}$, at zero when modeling the NuSTAR spectra, as the small amount of absorption toward MAXI J1820+070 does not affect energies above $3 \mathrm{keV}$.

The absorbed power-law model leaves systematic residuals, with a reduced chi-squared of $\chi^{2} / \nu_{\mathrm{EPOCH} 1}=1557.1 / 941=1.65$ and $\chi^{2} / \nu_{\text {ЕРОСН2 } 2}=1248.3 / 1127=1.11$. Reflection features are evident in the spectral residuals (see Figures 2(b) and (e)): an Fe $\mathrm{K} \alpha$ emission line is clearly detected by both NuSTAR and XMM-Newton centered around $6.4-6.5 \mathrm{keV}$, and a Compton reflection hump is evident in the NuSTAR spectra peaking around $30 \mathrm{keV}$. The prominent Compton reflection hump confirms that the $\mathrm{Fe} \mathrm{K} \alpha$ line originates from reflection by cold optically thick gas (e.g., Lightman \& White 1988). Accounting for the $\mathrm{Fe} \mathrm{K} \alpha$ line with a Gaussian model improves the fit by $\Delta \chi^{2}=384$ for the NuSTAR spectrum, and $\Delta \chi^{2}=88$ for XMM-Newton. The bestfit Gaussian model indicates that the $\mathrm{Fe} \mathrm{K} \alpha$ line is narrow, with a line width of $\sigma_{\mathrm{EPOCH} 1}=0.29_{-0.04}^{+0.05} \mathrm{keV}$ measured by NuSTAR and $\sigma_{\mathrm{EPOCH} 2}=0.24_{-0.06}^{+0.09} \mathrm{keV}$ measured by XMM-Newton, and the equivalent width (EW) is constrained to be $64 \pm 7 \mathrm{eV}$ and
$98_{-21}^{+20} \mathrm{eV}$ (see Table 1 for details). The line width and EW are significantly smaller than those measured with XMM-Newton EPIC-pn during the BHS $\left(\sigma_{\mathrm{BHS}} \approx 0.9 \mathrm{keV}\right.$ and $E W_{\mathrm{BHS}} \approx$ $270 \pm 30 \mathrm{eV}$; Kajava et al. 2019). The narrow and symmetric line profile we observe here indicates weak relativistic effects, which is direct evidence for the $\mathrm{Fe} \mathrm{K} \alpha$ line being produced far from the central black hole.

In order to physically model the reflection features, and to get a constraint of the disk truncation radius, we fit the spectra with the relxill relativistic disk reflection model (relxill v1.3.3, Dauser et al. 2014; García et al. 2014). We fix the disk emissivity indices, $q_{\text {in,out }}$ at 3 , the value expected for the outer part of a Shakura \& Sunyaev disk (Laor 1991; Dauser et al. 2013). This is a reasonable assumption, considering that the reflection features are produced at a large distance from the black hole. The emissivity indices cannot be constrained if left free. We fix the black hole spin, $a^{*}$, at the default value of 0.998 . This parameter is irrelevant here as the inner disk is truncated outside of the ISCO. The outer disk radius, $R_{\text {out }}$, is fixed at the maximum value of the model at $1000 r_{\mathrm{g}}\left(r_{\mathrm{g}} \equiv G M / c^{2}\right.$ is the gravitational radius). As the majority of the X-ray flux comes from the inner part of the accretion disk, the spectral modeling is not sensitive to $R_{\text {out }}$. The relxill model includes a coronal illuminating continuum in the shape of a power law with an exponential 
Table 1

Energy Spectral Parameters of MAXI J1820+070 in the LHS

\begin{tabular}{|c|c|c|}
\hline Parameter & EPOCH 1 & EPOCH 2 \\
\hline \multicolumn{3}{|c|}{ Fe K $\alpha$ line modeled with Gaussian: TBabs* (powerlaw+Gauss) } \\
\hline$N_{\mathrm{H}}\left(\times 10^{21} \mathrm{~cm}^{-2}\right)$ & .. & $0.93 \pm 0.03$ \\
\hline$E_{\mathrm{Fe}}(\mathrm{keV})$ & $6.37 \pm 0.04$ & $6.54 \pm 0.06$ \\
\hline$\sigma_{\mathrm{Fe}}(\mathrm{keV})$ & $0.29_{-0.04}^{+0.05}$ & $0.24_{-0.06}^{+0.09}$ \\
\hline $\mathrm{EW}_{\mathrm{Fe}}(\mathrm{eV})$ & $64 \pm 7$ & $98_{-21}^{+20}$ \\
\hline$\Gamma$ & $1.667 \pm 0.006$ & $1.799 \pm 0.006$ \\
\hline$\chi^{2} / \nu$ & $1173.4 / 939=1.25$ & $1160.3 / 1124=1.03$ \\
\hline \multicolumn{3}{|c|}{ Best-fit model: TBabs*relxill } \\
\hline$N_{\mathrm{H}}\left(\times 10^{21} \mathrm{~cm}^{-2}\right)$ & $\cdots$ & $1.03 \pm 0.04$ \\
\hline$q$ & $3^{f}$ & $3^{f}$ \\
\hline$\Gamma$ & $1.660_{-0.007}^{+0.008}$ & $1.765 \pm 0.008$ \\
\hline$E_{\text {cut }}(\mathrm{keV})$ & $>697$ & $>328$ \\
\hline$i\left(^{\circ}\right)$ & $<20$ & $<39$ \\
\hline$R_{\text {in }}\left(r_{\mathrm{g}}\right)$ & $27_{-6}^{+10}$ & $>38$ \\
\hline $\log (\xi)\left(\log \left(\mathrm{erg} \mathrm{cm} \mathrm{s}^{-1}\right)\right)$ & $2.99_{-0.17}^{+0.02}$ & $3.08 \pm 0.06$ \\
\hline$A_{\mathrm{Fe}}$ (solar) & $3.0_{-0.5}^{+0.6}$ & $6 \pm 2$ \\
\hline$R_{\text {ref }}$ & $0.060_{-0.007}^{+0.005}$ & $0.06 \pm 0.01$ \\
\hline$\chi^{2} / \nu$ & $1033.8 / 936=1.10$ & $1135.4 / 1121=1.01$ \\
\hline$F_{2-10 \mathrm{keV}}\left(\mathrm{erg} \mathrm{cm}^{-2} \mathrm{~s}^{-1}\right)$ & $5.0 \times 10^{-10}$ & $3.9 \times 10^{-11}$ \\
\hline$L_{0.1-100 \mathrm{keV}}\left(\mathrm{erg} \mathrm{s}^{-1}\right)$ & $2.6 \times 10^{36}$ & $1.9 \times 10^{35}$ \\
\hline$L_{\text {Edd }}(\%)$ & 0.25 & 0.018 \\
\hline
\end{tabular}

Note. Fixed parameters are marked with the superscript $f$. In this work, we adopt the distance estimate of $3 \mathrm{kpc}$ and black hole mass estimate of $8 M_{\odot}$ when calculating the source luminosity and Eddington ratio of MAXI J1820+070

cutoff at high energies, parameterized by the power-law index, $\Gamma$, and the high energy cutoff, $E_{\text {cut }}$. Other free model parameters are the inner disk radius, $R_{\mathrm{in}}$, the disk inclination, $i$, the ionization parameter, $\xi$, the iron abundance, $A_{\mathrm{Fe}}$, and the reflection fraction, $R_{\text {ref }}$.

The model, TBabs*relxill in XSPEC notation, describes the data well, leaving no systematic structures in the residuals with $\chi^{2} / \nu_{\text {ЕPOCH } 1}=1033.8 / 936=1.10$ and $\chi^{2} / \nu_{\text {EPOCH} 2}=1135.4 / 1122=1.01$ (see Figures $2(\mathrm{c})$ and (f)). We measure a low absorption column density, $N_{\mathrm{H}}=(1.03 \pm 0.04) \times 10^{21} \mathrm{~cm}^{-2}$ with XMM-Newton, consistent with the value obtained early on during the outburst (Shidatsu et al. 2018; Kajava et al. 2019). The spectral continuum is well described by a power law with a hard photon index of $\Gamma \approx 1.6-1.8$, with no prominent high energy cutoff required (see Table 1 for best-fit parameters). The observed flux of MAXI J1820+070 in 2-10 keV decreased from $5.0 \times 10^{-10}$ to $3.9 \times 10^{-11} \mathrm{erg} \mathrm{cm}^{-2} \mathrm{~s}^{-1}$ between EPOCH 1 and EPOCH 2, corresponding to a change in Eddington ratio from $0.35 \%$ to $0.026 \%$. Adding an extra thermal disk component modeled by diskbb does not improve the fit, implying that thermal emission from the accretion disk is either too weak to be detected, or the disk is sufficiently cool that the peak of the disk blackbody distribution moves below the XMM-Newton band.

The fitting results indicate that the disk is moderately ionized, with an ionization parameter of $\log (\xi) \approx 3$. We measure the truncation radius of the optically thick accretion disk to be $R_{\mathrm{in}, \mathrm{EPOCH} 1}=27_{-6}^{+10} r_{\mathrm{g}}$, and $R_{\mathrm{in}, \mathrm{EPOCH} 2}>38 r_{\mathrm{g}}$.
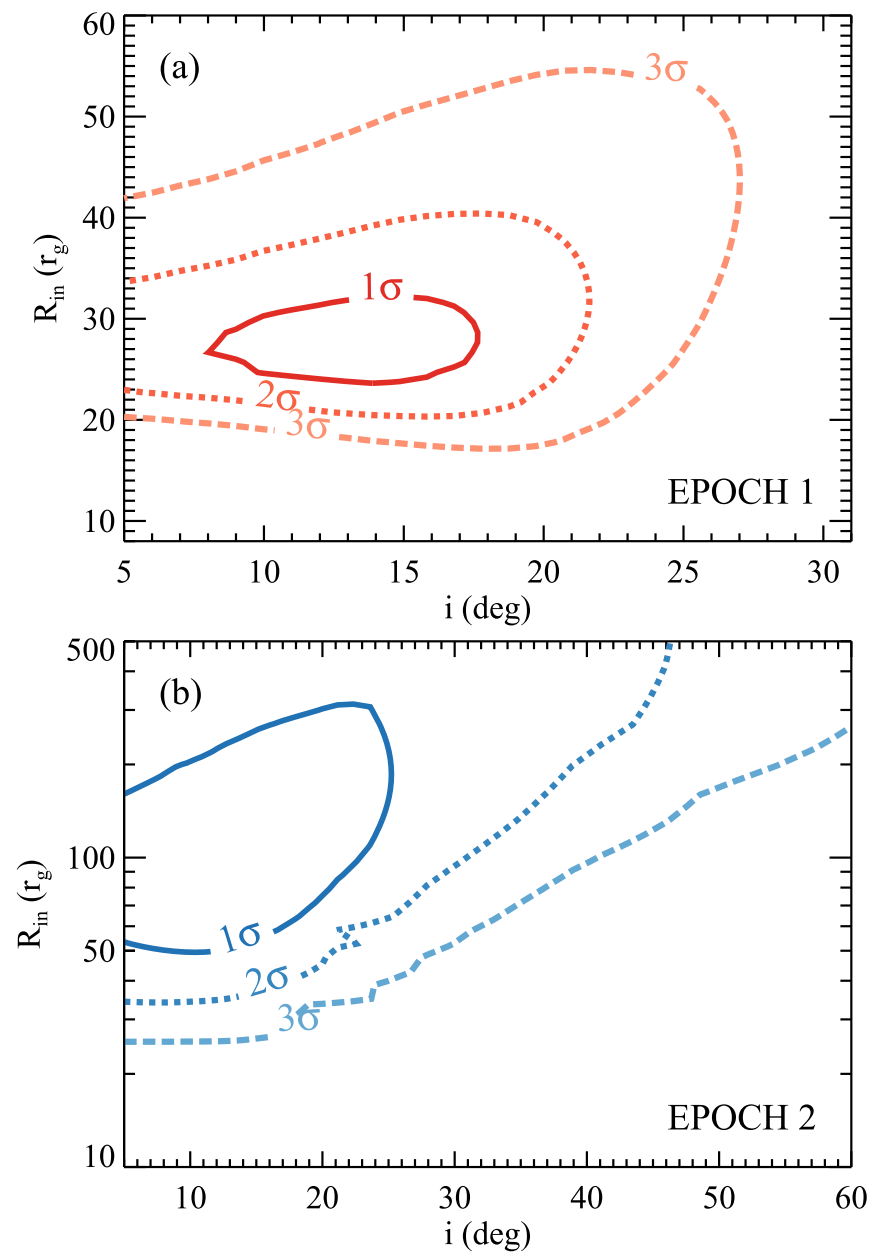

Figure 3. Confidence contours of the inner disk radius, $R_{\text {in }}$, and the disk inclination, $i$. $1 \sigma, 2 \sigma$, and $3 \sigma$ correspond to $\Delta \chi^{2}$ of 1 , 4 , and 9 , respectively.

Based on the definition of the ionization parameter, ${ }^{7}$ we estimate the density of the accretion disk, $n$, at the radius where the $\mathrm{Fe} \mathrm{K}$ line is generated, decreases from $\sim 10^{18}$ to $\sim 10^{16}$ $\mathrm{cm}^{-3}$ from EPOCH 1 to EPOCH 2. The best fit prefers a low inclination angle of the accretion disk, and only upper limits are obtained: $i_{\mathrm{EPOCH} 1}<20^{\circ}$ and $i_{\mathrm{EPOCH} 2}<39^{\circ}$. This is expected given the presence of a narrow $\mathrm{Fe} \mathrm{K} \alpha$ line, as line broadening caused by relativistic effects would become less apparent when the disk is viewed close to face-on (Laor 1991). $R_{\text {in }}$ and $i$ are degenerate, as they are both related to the width of the Fe $\mathrm{K} \alpha$ line. Without a more complicated line profile like the broad and asymmetric lines detected during the BHS, the two parameters cannot be uniquely constrained. To investigate the correlation between $R_{\text {in }}$ and $i$, we plot the $\Delta \chi^{2}$ contour in Figure 3 . As shown in panel (b), similar to case of GX 339-4 discussed in Tomsick et al. (2009), the model tends to prefer a larger inner disk radius with increasing disk inclination. Therefore, we note that by letting both $R_{\text {in }}$ and $i$ vary freely, we are quoting a conservatively small value for the disk truncation radius.

In addition, the best fit leads to a supersolar iron abundance, similar to that found in the BHS (Bharali et al. 2019; Buisson et al. 2019). It is currently uncertain whether the high iron abundance, frequently found when performing spectral

\footnotetext{
$\xi$ is defined as $\xi=L_{\mathrm{x}} /\left(n r^{2}\right)$, where $L_{\mathrm{x}}$ is the X-ray luminosity, $r$ is the distance between the gas and the $\mathrm{X}$-ray source, and $n$ is the gas density (hydrogen nucleus density).
} 
modeling with ionized disk reflection models, represents the true elemental abundance in the accretion disk, or is an overestimation resulting from physical processes overlooked in the calculation of the reflection models. There is evidence that this issue might be mitigated by using reflection models assuming high disk density (e.g., Tomsick et al. 2018; Jiang et al. 2019). We note that the iron abundance is known to be mostly related to the line strength rather than the line width, thus it is unlikely to have a significant effect on the estimate of the disk truncation radius here.

The best-fit reflection fraction is $R_{\text {ref }} \approx 0.06$, significantly lower than that measured in the BHS of BHBs, which often requires a reflection fraction greater than unity. This reflection fraction in the BHS is believed to be enhanced by strong lightbending effects near the black hole (e.g., Miniutti \& Fabian 2004; Reis \& Miller 2013; Xu et al. 2018a, 2018b). The reflection fraction parameter in the relxill model is defined as the ratio of the coronal intensity illuminating the disk to that reaching the observer. The extremely low value we find here in the LHS of MAXI J1820+070 indicates that solid angle extended by the reflector is small, which is consistent with the scenario that the inner accretion disk is significantly truncated.

\section{Timing Analysis}

We produced the power spectral density (PSD) from the NuSTAR (EPOCH 1) and XMM-Newton EPIC-pn (EPOCH 2) light curves, in the energy bands of 3-79 and $0.6-10 \mathrm{keV}$. The NuSTAR light curves of FPMA and FPMB are added using the lcmath tool in XRONOS. We produce the PSD from light curves with the time bins of $0.5 \mathrm{~s}$, averaged in intervals of $2^{13}$ bins. The PSD is calculated in the rms normalization using powspec, with white noise subtracted. The NuSTAR and XMM-Newton PSD is geometrically rebinned by a factor of 1.03 and 1.05 , respectively, to reach nearly equally spaced frequency bins in logarithmic scale. We fit the PSD in XSPEC with a multi-Lorentzian model using a unity response file: several zero-centered broad Lorentzians for the band-limited noise continuum, one narrow Lorentzian for the QPO and one for its possible subharmonic.

As shown in Figure 4, we find a QPO in the NuSTAR and $\mathrm{XMM}-$ Newton PSD at the frequency of $\nu_{\mathrm{EPOCH} 1}=11.6 \pm 0.2$ $\mathrm{mHz}$ and $\nu_{\mathrm{EPOCH} 2}=2.8 \pm 0.1 \mathrm{mHz}$, detected at $5.3 \sigma$ and $3.2 \sigma$ via the F-test. The QPO has rms variability of $13 \% \pm 1 \%$ and $11 \% \pm 3 \%$ in EPOCH 1 and EPOCH 2, respectively. The QPO is detected in the $\mathrm{mHz}$ range, lower than the typical frequency range of low-frequency QPOs found in BHBs $(0.1-30 \mathrm{~Hz})$. But the shape of the noise continuum and the fact that QPO is located close to the low-frequency break are consistent with type-C QPOs commonly found in BHBs (Belloni \& Motta 2016). The PSD is similar to that detected in MAXI $\mathrm{J} 1820+070$ during the BHS (Buisson et al. 2019), only with the QPO and the low-frequency break extending to even lower frequencies in the LHS.

\section{Discussion and Conclusion}

We detect a narrow $\mathrm{Fe} \mathrm{K} \alpha$ emission line with high $\mathrm{S} / \mathrm{N}$ from NuSTAR and XMM-Newton observations of the black hole X-ray binary MAXI J1820+070 during the second rebrightening period after its 2018 main outburst. The X-ray spectral and timing properties indicate that the source was in the LHS during the time of the observations. Spectral modeling reveals a



Figure 4. NuSTAR and XMM-Newton PSD of MAXI J1820+070 in the LHS. The best-fit models and their individual Lorentzian components are marked in solid and dashed lines.

very low absorption column density, combined with the moderate ionization state of the reflection material, confirming that the $\mathrm{Fe} \mathrm{K} \alpha$ line is produced from reflection by the accretion disk rather than that by torus-like Compton thick obscuring material commonly found in AGNs (Hickox \& Alexander 2018) and in the BHB V404 Cygni (Motta et al. 2017). The line is visibly narrow and lacks significant relativistic broadening, in contrast to the broad line profile observed during the BHS (see Figure 5(a) for a comparison of the line profile ${ }^{8}$ ), providing direct evidence for significant truncation of the inner accretion disk at low accretion rates in a BHB.

There are disparities in the literature about the estimate of the disk inclination in MAXI J1820+070. X-ray dips were observed during early phases of the outburst (Kajava et al. 2019), and a sharp increase in the $\mathrm{H} \alpha$ emission line $\mathrm{EW}$ was reported and interpreted as a grazing eclipse of the accretion disk (Torres et al. 2019), suggesting a high inclination of $i \approx 60^{\circ}-80^{\circ}$ for the outer part of the accretion disk. We note that the inclination of this system is also unlikely to be very low (e.g., $<10^{\circ}$ ) because radial velocity measurements are significant in amplitude (Torres et al. 2019). The measured jet inclination angle of $62^{\circ} \pm 3^{\circ}$ also indicates that the system is viewed at high inclination (Atri et al. 2020). In contrast, modeling the relativistic reflection spectra during the BHS yields a low inclination of $i \approx 30^{\circ}$ for the inner part of the accretion disk (Bharali et al. 2019; Buisson et al. 2019). If both inclination estimates are robust, this implies a strong disk warp of $\sim 30^{\circ}-50^{\circ}$. Our spectral fitting of the narrow $\mathrm{Fe} \mathrm{K} \alpha$ line in the LHS also prefers a very low inclination. However, as discussed above, it is

\footnotetext{
8 We choose the representative Fe K $\alpha$ line profile of MAXI J1820+070 in the BHS from the NuSTAR observation on 2018 March 21 (ObsID: 90401309006), detailed analysis of this data set is published in Buisson et al. (2019).
} 

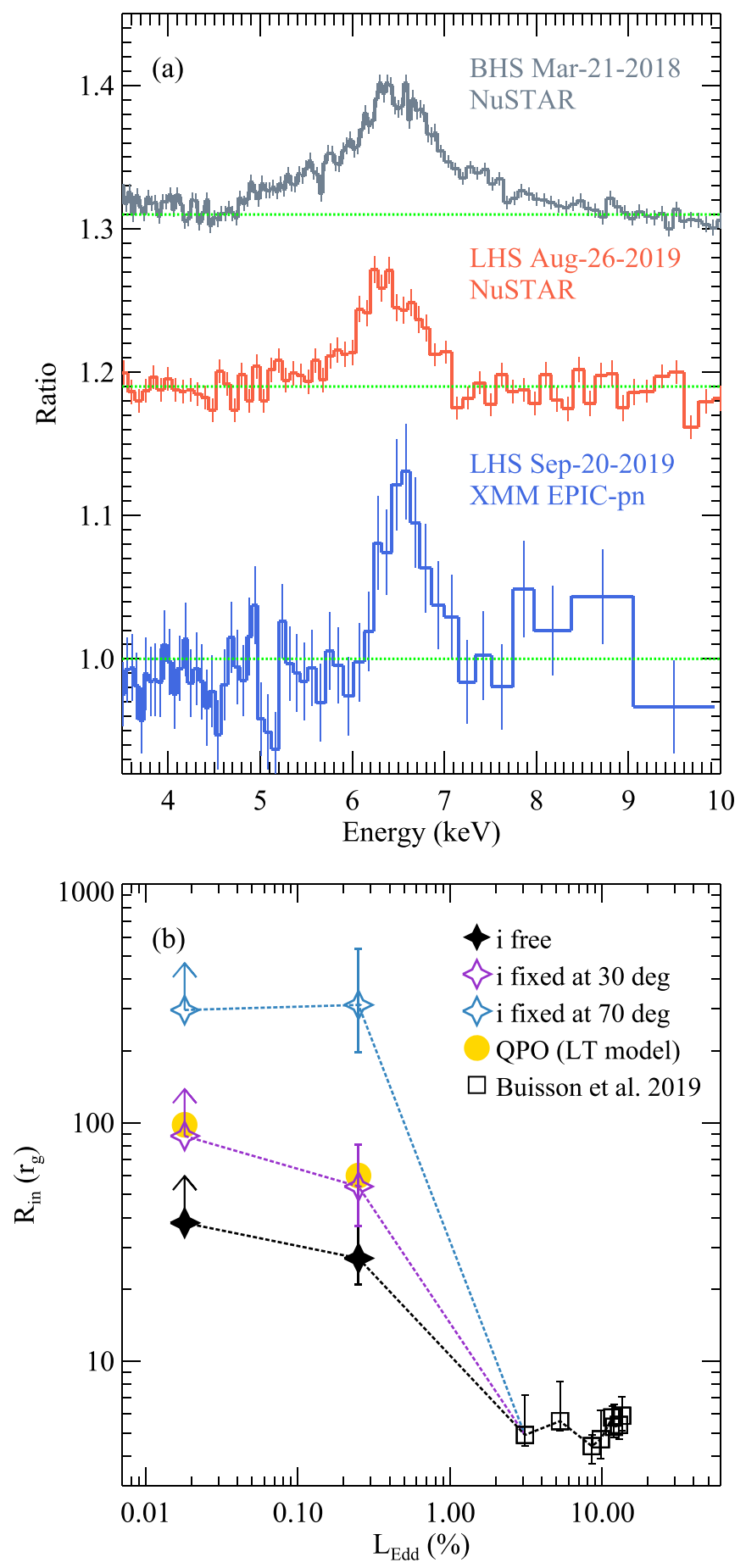

Figure 5. (a) A comparison of the $\mathrm{Fe} \mathrm{K} \alpha$ line profile of MAXI J1820+070 observed at different epochs. The $\mathrm{Fe} \mathrm{K} \alpha$ becomes visually narrower during the LHS when compared that during the BHS (Buisson et al. 2019). We caution that the apparent difference in the narrow line profile observed by NuSTAR and XMM-Newton during the LHS is at least partially due to the different energy resolution of the instruments, which is about $400 \mathrm{eV}$ for NuSTAR and $100 \mathrm{eV}$ for EPIC-pn on XMM-Newton. (b) Evolution of the inner accretion disk radius with the Eddington ratio measured in MAXI J1820+070.

expected for the spectral modeling to bias toward low inclinations in the presence of a narrow line profile. Without strong relativistic distortion effects, the inclination is poorly constrained as only upper limits are obtained. Therefore, we tried fitting the spectra with a fixed disk inclination angle instead, and still assuming a disk emissivity index of $q=3$. This results in a larger disk truncation radius and slightly degraded quality of the fits: when $i$ is frozen at $30^{\circ}$, we get $R_{\text {in,EPOCH1 }}=54_{-17}^{+27} r_{\mathrm{g}}$ and $R_{\text {in, ЕРOCH2 }}>88 r_{\mathrm{g}}$ with $\Delta \chi_{\mathrm{EPOCH} 1}^{2}=11.7$ and $\Delta \chi_{\mathrm{EPOCH} 2}^{2}=1.6$; when $i$ is fixed at $70^{\circ}$, the constraint on the inner disk radius becomes $R_{\text {in,EPOCH1 }}=312_{-114}^{+226} r_{\mathrm{g}}$ and $R_{\mathrm{in}, \mathrm{EPOCH} 2}>297 r_{\mathrm{g}}$ with $\Delta \chi_{\mathrm{EPOCH} 1}^{2}=9.9$ and $\Delta \chi_{\mathrm{EPOCH} 2}^{2}=5.2$. These fits, although statistically slightly worse, leave no clear residuals with physical implications, thus may still be considered acceptable within calibration uncertainties.

There have been a number of observational campaigns aimed at investigating the evolution of the inner disk radius with the accretion rate in BHBs via the reflection method (e.g., Petrucci et al. 2014; Fürst et al. 2016). However, the results are often poorly constrained or highly model dependent due to low statistics, especially at low flux states. In this work, we find evidence for a large inner disk radius in MAXI J1820+070 at low accretion rates (a few tens to a few hundreds of $r_{\mathrm{g}}$, depending on the disk inclination). Combined with earlier measurements during the BHS by NuSTAR (Bharali et al. 2019; Buisson et al. 2019), it suggests that the inner edge of the accretion disk remains stable around the ISCO when the accretion rate is high, and starts to recede from the ISCO as the luminosity drops below $\sim 1 \%$ of the Eddington luminosity (see Figure 5(b)). The critical accretion rate when significant disk truncation occurs is consistent with that measured in the well-studied source GX 339-4 via the reflection method (Tomsick et al. 2009), and that found based on the study of several BHBs by systematically modeling their thermal disk components (Cabanac et al. 2009). In terms of the disk recessing with accretion rate, the results agree well with the theoretical prediction that the inner part of accretion disk becomes replaced by ADAF at low accretion rates $(<1 \%$ of the Eddington limit), although the model predicts that the optically thick accretion disk should be truncated in all hard states (Esin et al. 1997).

In addition, we detected a QPO at $\nu_{\mathrm{EPOCH} 1}=11.6 \pm 0.2$ $\mathrm{mHz}$ and $\nu_{\mathrm{EPOCH} 2}=2.8 \pm 0.1 \mathrm{mHz}$. The QPO and lowfrequency break are found at lower frequencies than those in the BHS. Qualitatively, the longer timescales imply a physically larger size for the hot optically thin Comptonization regions around black holes, where the QPO is believed to be generated. In the propagating mass accretion rate fluctuations model, the low-frequency break marks the viscous timescale at the outer edge of the Comptonization region (e.g., Ingram \& Done 2010; Ingram \& van der Klis 2013). There have been various theoretical models put forward to explain the lowfrequency QPOs in BHBs, but the exact mechanism is still highly uncertain. One of the currently promising models is the Lense-Thirring (LT) precession model. Adopting the simplified assumption that the QPO is caused by the effect of the LT precession of a test particle orbiting a spinning black hole at the disk truncation radius (Motta et al. 2018), we calculate the inner disk radius inferred from the QPO frequencies using the black hole mass of $8 M_{\odot}$ and the spin of $a^{*}=0.3$. This leads to the characteristic truncation radius of $R_{\text {in, EPOCH1 }} \sim 60 r_{\mathrm{g}}$ and $R_{\text {in,EPOCH2 }} \sim 100 r_{\mathrm{g}}$. As a crude estimate, these are broadly similar to the spectral modeling results, in support of the inner accretion disk being significantly truncated in the LHS. During the BHS, however, we note that there is usually disagreement in the measurements from the two methods (e.g., Fürst et al. 2016; Xu et al. 2017; Buisson et al. 2019), especially, Buisson et al. (2019) find that the QPO frequency and the disk inner 
radius are not connected. As discussed in Ingram \& Done (2011), it is possible that the discrepancy is related to physical complexities currently not well understood and thus not included in the QPO models, or complexities related to intrinsic properties of the corona.

Additional uncertainties in the measurement of the inner disk radius come from the poorly-known nature of the corona, which affects the illumination pattern of disk (Fabian et al. 2014). During the above analysis, we assume the disk emissivity of $\epsilon \propto r^{-q}(q=3)$, which is expected for a standard accretion disk in the Newtonian regime. An alternative explanation for the narrow $\mathrm{Fe} \mathrm{K}$ line that does not involve disk truncation is a low disk emissivity index $(q<2)$, so that most of the contribution to the reflection features come from the outer disk. One possible accretion geometry that yields such a low emissivity index is a large lamppost height for the corona, which is believed to be associated with the base of a jet (Dauser et al. 2013). The emissivity expected for a lamppost geometry in Newtonian gravity is $q \sim 0$ for $r<h$, and $q \sim 3$ for $r>h$ (Vaughan et al. 2004). The spectra can be equally well fitted by the reflection model assuming a lamppost geometry, relxilllp, with a lamppost height of $h_{\mathrm{EPOCH} 1} \sim 40 r_{\mathrm{g}}$ and $h_{\mathrm{EPOCH} 2}>70 r_{\mathrm{g}}$, without the need to invoke disk truncation. But the model cannot self-consistently explain the low reflection fraction, unless significant beaming away from the accretion disk is involved. For a reflection fraction of $\sim 0.1$, it requires bulk motion with a Lorentz factor of $\gamma \sim 1.2$ when viewed at the inclination of $30^{\circ}$, and $\gamma \sim 1.6$ at the inclination of $60^{\circ}$ (Beloborodov 1999). It is uncertain whether strong beaming and the accretion geometry of a compact corona with a large lamppost height above the accretion disk are realistic descriptions of the system. There is evidence that significant beaming is absent in X-ray emission of BHBs in the LHS (Narayan \& McClintock 2005). Although the case at low accretion rates is less clear, previous successful applications of the lamppost model to X-ray spectra of bright BHBs and AGNs measures a low lamppost height of $<10 r_{\mathrm{g}}$, or a steep disk emissivity profile (Fabian et al. 2015). Thus the physical implications of such a large lamppost height or low emissivity index required by the spectral fitting here are currently unclear and requires further investigation. The extended diskcorona model and the lamppost corona model are two competing coronal geometries that have been proposed (Chauvin et al. 2018). The QPO frequency and low-frequency break in the PSD suggest that a physically large size for the Comptonization region, consistent with the extended corona and receding accretion disk scenario. The inner disk being truncated at a large radius also naturally explains the nondetection of any thermal disk emission. Disk truncation provides a straightforward and physically reasonable explanation for the narrow $\mathrm{Fe}$ $\mathrm{K} \alpha$ line we detected in MAXI J1820+070. However, we note that an alternative accretion geometry of a high lamppost corona cannot be ruled out by our data set and may also be plausible.

We thank the referee for helpful comments that improved the paper. We thank Norbert Schartel for approving the XMMNewton DDT observation and the XMM-Newton SOC for prompt scheduling of the observation. J.H. acknowledges support from an appointment to the NASA Postdoctoral Program at the Goddard Space Flight Center, administered by the USRA through a contract with NASA. D.J.W. acknowledge support from an STFC Ernest Rutherford Fellowship. This work was supported under NASA contract No. NNG08FD60C and made use of data from the NuSTAR mission, a project led by the California Institute of Technology, managed by the Jet Propulsion Laboratory, and funded by the National Aeronautics and Space Administration. We thank the NuSTAR Operations, Software, and Calibration teams for support with the execution and analysis of these observations.

\section{ORCID iDs}

Yanjun Xu (iD https://orcid.org/0000-0003-2443-3698 Fiona A. Harrison (i) https://orcid.org/0000-0003-2992-8024

John A. Tomsick (iD https://orcid.org/0000-0001-5506-9855 Jeremy Hare (iD https://orcid.org/0000-0002-8548-482X Andrew C. Fabian (iD https://orcid.org/0000-0002-9378-4072 Dominic J. Walton (iD https://orcid.org/0000-0001-5819-3552

\section{References}

Arnaud, K. A. 1996, in ASP Conf. Ser. 101, Astronomical Data Analysis Software and Systems V, ed. G. H. Jacoby \& J. Barnes (San Francisco, CA: ASP), 17

Atri, P., Miller-Jones, J. C. A., Bahramian, A., et al. 2020, MNRAS, 493, L81 Barthelmy, S. D., Barbier, L. M., Cummings, J. R., et al. 2005, SSRv, 120, 143 Belloni, T. M., \& Motta, S. E. 2016, in Astrophysics of Black Holes, ed. C. Bambi (Berlin: Springer), 61

Beloborodov, A. M. 1999, ApJL, 510, L123

Bharali, P., Chauhan, J., \& Boruah, K. 2019, MNRAS, 487, 5946

Bright, J., Fender, R., \& Motta, S. 2018, ATel, 11420, 1

Buisson, D. J. K., Fabian, A. C., Barret, D., et al. 2019, MNRAS, 490, 1350 Burrows, D. N., Hill, J. E., Nousek, J. A., et al. 2005, SSRv, 120, 165

Cabanac, C., Fender, R. P., Dunn, R. J. H., \& Körding, E. G. 2009, MNRAS, 396, 1415

Chauvin, M., Florén, H. G., Friis, M., et al. 2018, NatAs, 2, 652

Corral-Santana, J. M., Casares, J., Muñoz-Darias, T., et al. 2016, A\&A, 587, A61

Dauser, T., García, J., Parker, M. L., Fabian, A. C., \& Wilms, J. 2014, MNRAS, 444, L100

Dauser, T., Garcia, J., Wilms, J., et al. 2013, MNRAS, 430, 1694

Esin, A. A., McClintock, J. E., \& Narayan, R. 1997, ApJ, 489, 865

Fabian, A. C., Lohfink, A., Kara, E., et al. 2015, MNRAS, 451, 4375

Fabian, A. C., Parker, M. L., Wilkins, D. R., et al. 2014, MNRAS, 439, 2307

Fabian, A. C., Rees, M. J., Stella, L., \& White, N. E. 1989, MNRAS, 238, 729

Fürst, F., Tomsick, J. A., Yamaoka, K., et al. 2016, ApJ, 832, 115

Gaia Collaboration, Brown, A. G. A., Vallenari, A., et al. 2018, A\&A, 616, A1

Gandhi, P., Rao, A., Johnson, M. A. C., Paice, J. A., \& Maccarone, T. J. 2019, MNRAS, 485, 2642

García, J. A., Dauser, T., Lohfink, A., et al. 2014, ApJ, 782, 76

Harrison, F. A., Craig, W. W., Christensen, F. E., et al. 2013, ApJ, 770, 103

Hickox, R. C., \& Alexander, D. M. 2018, ARA\&A, 56, 625

Hoang, J., Molina, E., Lopez, M., et al. 2019, Proc. ICRC (Madison, WI), 36,696

Ingram, A., \& Done, C. 2010, MNRAS, 405, 2447

Ingram, A., \& Done, C. 2011, MNRAS, 415, 2323

Ingram, A., \& van der Klis, M. 2013, MNRAS, 434, 1476

Jansen, F., Lumb, D., Altieri, B., et al. 2001, A\&A, 365, L1

Jiang, J., Fabian, A. C., Wang, J., et al. 2019, MNRAS, 484, 1972

Kajava, J. J. E., Motta, S. E., Sanna, A., et al. 2019, MNRAS, 488, L18

Kara, E., Steiner, J. F., Fabian, A. C., et al. 2019, Natur, 565, 198

Kawamuro, T., Negoro, H., Yoneyama, T., et al. 2018, ATel, 11399

Krimm, H. A., Holland, S. T., Corbet, R. H. D., et al. 2013, ApJS, 209, 14

Laor, A. 1991, ApJ, 376, 90

Lightman, A. P., \& White, T. R. 1988, ApJ, 335, 57

Meyer, F., Liu, B. F., \& Meyer-Hofmeister, E. 2000, A\&A, 361, 175

Miller, J. M. 2007, ARA\&A, 45, 441

Miller, J. M., Tomsick, J. A., Bachetti, M., et al. 2015, ApJL, 799, L6

Miniutti, G., \& Fabian, A. C. 2004, MNRAS, 349, 1435

Motta, S. E., Franchini, A., Lodato, G., \& Mastroserio, G. 2018, MNRAS, 473, 431

Motta, S. E., Kajava, J. J. E., Sánchez-Fernández, C., Giustini, M., \& Kuulkers, E. 2017, MNRAS, 468, 981

Narayan, R., \& McClintock, J. E. 2005, ApJ, 623, 1017

Paice, J. A., Gandhi, P., Shahbaz, T., et al. 2019, MNRAS, 490, L62 
Petrucci, P. O., Cabanac, C., Corbel, S., Koerding, E., \& Fender, R. 2014, A\&A, 564, A37

Reis, R. C., \& Miller, J. M. 2013, ApJL, 769, L7

Remillard, R. A., \& McClintock, J. E. 2006, ARA\&A, 44, 49

Shidatsu, M., Nakahira, S., Yamada, S., et al. 2018, ApJ, 868, 54

Strüder, L., Briel, U., Dennerl, K., et al. 2001, A\&A, 365, L18

Taam, R. E., Liu, B. F., Yuan, W., \& Qiao, E. 2012, ApJ, 759, 65

Tomsick, J. A., Parker, M. L., García, J. A., et al. 2018, ApJ, 855, 3

Tomsick, J. A., Yamaoka, K., Corbel, S., et al. 2009, ApJL, 707, L87

Torres, M. A. P., Casares, J., Jiménez-Ibarra, F., et al. 2019, ApJL, 882, L21

Tucker, M. A., Shappee, B. J., Holoien, T. W. S., et al. 2018, ApJL, 867, L9
Vaughan, S., Fabian, A. C., Ballantyne, D. R., et al. 2004, MNRAS, 351, 193 Verner, D. A., Ferland, G. J., Korista, K. T., \& Yakovlev, D. G. 1996, ApJ, 465,487

Walton, D. J., Tomsick, J. A., Madsen, K. K., et al. 2016, ApJ, 826, 87

Wilms, J., Allen, A., \& McCray, R. 2000, ApJ, 542, 914

Xu, Y., García, J. A., Fürst, F., et al. 2017, ApJ, 851, 103

Xu, Y., Harrison, F., \& Tomsick, J. 2019, ATel, 13025, 1

Xu, Y., Harrison, F. A., García, J. A., et al. 2018a, ApJL, 852, L34

Xu, Y., Harrison, F. A., Kennea, J. A., et al. 2018b, ApJ, 865, 18

Yu, W., Zhang, J., Yan, Z., Wang, X., \& Bai, J. 2018, ATel, 11510, 1

Yuan, F., \& Narayan, R. 2014, ARA\&A, 52, 529

Zhang, S. N., Cui, W., \& Chen, W. 1997, ApJL, 482, L155 